\title{
4. Regionaler Forschungskongress der Psychiatrischen und Neurologischen Fachkrankenhäuser Bayerns am 21.-23.10.2003, Irsee
}

Helmfried E. Klein

In dieser Ausgabe der Psychiatrischen Praxis erscheinen 64 Artikel aus Fachkrankenhäusern mit psychiatrischen und neurologischen Kliniken, also Einrichtungen, die sich nicht traditionell als forschende und lehrende Institutionen verstehen. Dies bedarf einer Erklärung. Durch die traditionell enge Kooperation zwischen den bayerischen Fachkrankenhäusern und durch einen, in den letzten Jahren regelmäßig stattfindenden, wissenschaftlichen Austausch im Rahmen der regionalen Tagungen im Bildungszentrum Irsee wurde dieses beachtliche Forschungspotenzial aus diesen Versorgungskrankenhäusern gefördert.

Die hier berichteten Forschungsaktivitäten aus den Fachkrankenhäusern haben naturgemäß ihren Schwerpunkt in der anwendungsorientierten Forschung. Versorgungskrankenhäuser bieten für dieses Forschungssegment besonders gute Voraussetzungen. Die bisherigen Publikationen aus der Forschung von Fachkrankenhäusern fanden eine beachtliche Resonanz; dies zeigte sich nicht zuletzt in der Entwicklung der Zitierhäufigkeit dieser Zeitschrift. Forschung aus traditioneller Quelle, wie den Universitäten und Max-Planck-Instituten wird häufig kritisch entgegengebracht, sie sei durch den dort gegebenen Wettbewerb und durch den Karrieredruck - „publish or perrish“ zu kurzatmig und zu wenig an den Bedürfnissen der Patienten orientiert. Die grundsätzlich anderen Rahmenbedingungen für Forschung an Versorgungskrankenhäusern dürften dazu beitragen, dass Forschungsergebnisse publiziert werden, die so großen Anklang finden.

Sehr erfreulich ist auch die wachsende Zahl der Beiträge aus dem Fachbereich der Neurologie. Die Forschungsfelder von Psychiatrie und Neurologie rücken umso näher aneinander, je weiter sich die beiden Fachbereiche zu den aktuellen Forschungsfronten hinbewegen. Im Bereich z. B. der Neurodegeneration, der mo- lekularen Genetik, der Neuroendokrinologie und der Neurophysiologie unterscheiden sich die Forschungsobjekte und die Forschungsmethoden zwischen Neurologie und Psychiatrie kaum mehr. Die Neurologen stimulieren Stammganglien Parkinsonkranker elektrophysiologisch, die Psychiater behandeln Depressive durch Vagusnervstimulationen. Die Neurologie nutzt elektromagnetische Felder, um nervale Leitungsbahnen funktionell zu prüfen, die Psychiatrie nutzt die elektromagnetischen Felder zur Neuromodulation bei Depression und bei Tinnituspatienten und beginnen die mittels elektromagnetischer Doppelimpulstechnik funktionelle Störungen im Thalamus bei schizophrenen Erkrankungen zu evaluieren. Unsere amerikanischen Fachkollegen haben das jährliche Meeting ihrer Fachgesellschaft kürzlich unter das Motto gestellt, „Mind Meets Brain“; sie haben damit treffender als man es in deutscher Sprache sagen könnte, den Konvergenzprozess von Psychiatrie und Neurologie hin zum gemeinsamen Feld der Neurowissenschaften thematisiert.

Im Beginn der medizinischen Ausbildung der meisten von uns war es noch rein hypothetisch, dass Fühlen, Denken, Wollen sich in Form fassbarer biologischer Prozesse und in umschriebenen, morphologisch definierbaren Substraten des ZNS abspielen würde. Wer sich damals zu dieser Hypothese bekannte, machte sich als ideologisch verbohrter Somatiker verdächtig. Funktionelles NMR, PET und SPECT ermöglichen es uns heute, dass das in der ICD-10 definierte Kriterium für Persönlichkeitsstörungen, „das herzlose Unbeteiligtsein an Gefühlen für andere“ sichtbar gemacht und abgebildet werden kann. Die „Decade Of The Brain“ war der Zeit von 1991 - 2001 gewidmet. Auch wenn dieses Jahrzehnt längst um ist, so ist doch auch für die weitere Zukunft der Forschungsperspektive für die Psychiatrie und für die Neurologie im gemeinsamen Feld der Neurowissenschaft eine Herausforderung von höchster Priorität.

\section{Korrespondenzadresse \\ Prof. Dr. Helmfried E. Klein · Klinik und Poliklinik für Psychiatrie und Psychotherapie der Universität Regensburg · Universitätsstraße 84 ·93053 Regensburg · E-mail: helmfried.klein@medbo.de}

